ESAIM: PROCEEDINGS, Juillet 2013, Vol. 40, p. 83-102

C. Bourdarias, S. Gerbi, Editors

\title{
EXPLICIT STAGGERED SCHEMES FOR THE COMPRESSIBLE EULER EQUATIONS
}

\author{
R. Herbin ${ }^{1}$, J.-C. Latché ${ }^{2}$ and T.T. NGUYen ${ }^{3}$
}

\begin{abstract}
We review in this paper an explicit scheme for the numerical simulation of inviscid compressible flows; we analyze it for both the barotropic Euler equations and the full Euler equations for an ideal gas. In each case, we summarize the theoretical results that were recently obtained concerning the stability and consistency of the schemes and present some numerical results which confirm their good performance.
\end{abstract}

\section{INTRODUCTION}

The objective pursued in this work is to develop and study, from a theoretical point of view, an explicit scheme for the simulation of non viscous compressible flows, modeled either by the barotropic Euler equations or by the full Euler equations for an ideal gas. Our basic choice is to use an explicit variant of implicit and semi-implicit schemes that were developed and studied recently in the framework of the simulation of compressible flows at all speeds $[6,10,11,13]$; in these latter works, the implicit scheme is studied as a first step in the mathematical analysis of pressure correction schemes, which extend algorithms that are classical in the incompressible framework; these are based on (inf-sup stable) staggered discretizations. In our approach, the upwinding techniques which are implemented for stability reasons are performed for each equation separately and with respect to the material velocity only. This is in contradiction with the most common strategy adopted for hyperbolic systems, where upwinding is built from the wave structure of the system (see e.g. [2, 22] for surveys). However, it yields algorithms which are used in practice (see e.g. the so-called AUSM family of schemes $[18,19]$ ), because of their generality (a closed-form solution of Riemann problems is not needed), their implementation simplicity and their efficiency, thanks to an easy construction of the fluxes at the cell faces. But these schemes are scarcely studied from a theoretical point of view; one of our main concerns here will thus be to bring, as far as possible, theoretical arguments supporting our numerical developments.

We give in this paper a review of the results obtained for the explicit version of the schemes in the case of the (inviscid) Euler equations, and refer to [12] for a review of the results of the implicit and semi-implicit versions, to $[10,11,15,16,20]$ for the detailed proofs of the results, and to [7] for the implementation of the pressure correction scheme in the case of a drift-diffusion model for two phase flows.

The paper is organized as follows. We start by the description of the staggered meshes which are used for the discretization in space, using either a finite volume - non-conforming finite element or a full "MAC-type"

\footnotetext{
1 Université d'Aix-Marseille, herbin@cmi.univ-mrs.fr

2 Institut de Radioprotection et de Sûreté Nucléaire, jean-claude.latche]@irsn.fr

${ }^{3}$ Institut de Radioprotection et de Sûreté Nucléaire, trung.nguyen@gmail.com
}

(C) EDP Sciences, SMAI 2013 
finite volume scheme. We then study the scheme for the barotropic Euler equations in Section 3, and for the full Euler equations in Section 4, and give some numerical results in Section 5.

\section{MESHES AND UNKNOWNS}

Let $\Omega$, the computational domain, be an open bounded connected subset of $\mathbb{R}^{d}, 1 \leq d \leq 3$, of boundary $\partial \Omega$. In this section, we focus on the definition of the space discretization in the multidimensional cases, the adaptation to the 1D case being straightforward (see Section 3.3).

Let $\mathcal{M}$ be a decomposition of $\Omega$, supposed to be regular in the usual sense of the finite element literature (e.g. [3]). The cells may be:

- for a general domain $\Omega$, either convex quadrilaterals $(d=2)$ or hexahedra $(d=3)$ or simplices, both type of cells being possibly combined in a same mesh,

- for a domain the boundaries of which are hyperplanes normal to a coordinate axis, rectangles $(d=2)$ or rectangular parallelepipeds $(d=3)$ (the faces of which, of course, are then also necessarily normal to a coordinate axis).

By $\mathcal{E}$ and $\mathcal{E}(K)$ we denote the set of all $(d-1)$-faces $\sigma$ of the mesh and of the element $K \in \mathcal{M}$ respectively. The set of faces included in the boundary of $\Omega$ is denoted by $\mathcal{E}_{\text {ext }}$ and the set of internal edges (i.e. $\mathcal{E} \backslash \mathcal{E}_{\text {ext }}$ ) is denoted by $\mathcal{E}_{\text {int }}$; a face $\sigma \in \mathcal{E}_{\text {int }}$ separating the cells $K$ and $L$ is denoted by $\sigma=K \mid L$. The outward normal vector to a face $\sigma$ of $K$ is denoted by $\boldsymbol{n}_{K, \sigma}$. For $K \in \mathcal{M}$ and $\sigma \in \mathcal{E}$, we denote by $|K|$ the measure of $K$ and by $|\sigma|$ the $(d-1)$-measure of the face $\sigma$. For $1 \leq i \leq d$, we denote by $\mathcal{E}^{(i)} \subset \mathcal{E}$ and $\mathcal{E}_{\text {ext }}^{(i)} \subset \mathcal{E}_{\text {ext }}$ the subset of the faces of $\mathcal{E}$ and $\mathcal{E}_{\text {ext }}$ respectively which are perpendicular to the $i^{t h}$ unit vector of the canonical basis of $\mathbb{R}^{d}$.

The space discretization is staggered, using either the Marker-And Cell (MAC) scheme [8,9], or nonconforming low-order finite element approximations, namely the Rannacher and Turek (RT) element [21] for quadrilateral or hexahedric meshes, or the lowest degree Crouzeix-Raviart (CR) element [4] for simplicial meshes.

For all these space discretizations, the degrees of freedom for the scalar variables (i.e. the discrete pressure and density unknowns in the barotropic case and the discrete pressure, density and internal energy unknowns for the Euler equations) are associated to the cells of the mesh $\mathcal{M}$. They are denoted by $\left\{p_{K}, \rho_{K}, K \in \mathcal{M}\right\}$ (barotropic case) or $\left\{p_{K}, \rho_{K}, e_{K}, K \in \mathcal{M}\right\}$ (Euler equations).

Let us then turn to the degrees of freedom for the velocity (i.e. the discrete velocity unknowns).

- Rannacher-Turek or Crouzeix-Raviart discretizations - The degrees of freedom for the velocity components are located at the center of the faces of the mesh, and we choose the version of the element where they represent the average of the velocity through a face. The set of degrees of freedom reads:

$$
\left\{u_{\sigma, i}, \sigma \in \mathcal{E}, 1 \leq i \leq d\right\} .
$$

- MAC discretization - The degrees of freedom for the $i^{t h}$ component of the velocity are defined at the centre of the faces of $\mathcal{E}^{(i)}$, so the whole set of discrete velocity unknowns reads:

$$
\left\{u_{\sigma, i}, \sigma \in \mathcal{E}^{(i)}, 1 \leq i \leq d\right\} .
$$

We now introduce a dual mesh, which will be used for the finite volume approximation of the time derivative and convection terms in the momentum balance equation.

- Rannacher-Turek or Crouzeix-Raviart discretizations - For the RT or CR discretizations, the dual mesh is the same for all the velocity components. When $K \in \mathcal{M}$ is a simplex, a rectangle or a cuboid, for $\sigma \in \mathcal{E}(K)$, we define $D_{K, \sigma}$ as the cone with basis $\sigma$ and with vertex the mass center of $K$ (see Figure 1 ). We thus obtain a partition of $K$ in $m$ sub-volumes, where $m$ is the number of faces of the mesh, each sub-volume having the same measure $\left|D_{K, \sigma}\right|=|K| / m$. We extend this definition to general quadrangles 


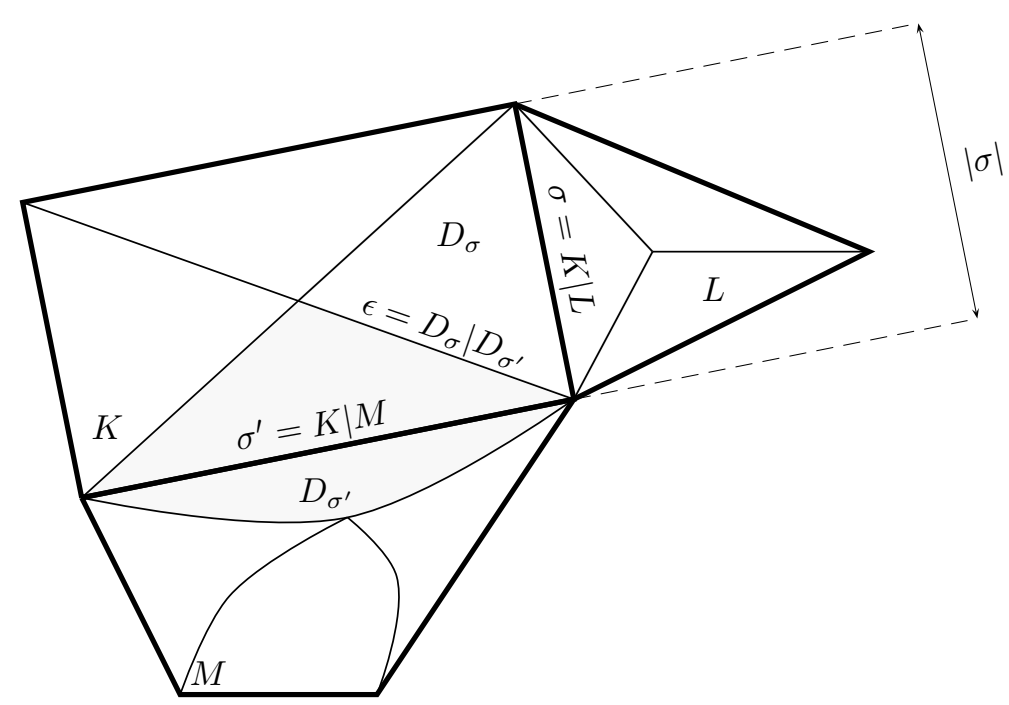

Figure 1. Primal and dual meshes for the Rannacher-Turek and Crouzeix-Raviart elements.

and hexahedra, by supposing that we have built a partition still of equal-volume sub-cells, and with the same connectivities; note that this is of course always possible, but that such a volume $D_{K, \sigma}$ may be no longer a cone; indeed, if $K$ is far from a parallelogram, it may not be possible to build a cone having $\sigma$ as basis, the opposite vertex lying in $K$ and a volume equal to $|K| / m$. The volume $D_{K, \sigma}$ is referred to as the half-diamond cell associated to $K$ and $\sigma$.

For $\sigma \in \mathcal{E}_{\text {int }}, \sigma=K \mid L$, we now define the diamond cell $D_{\sigma}$ associated to $\sigma$ by $D_{\sigma}=D_{K, \sigma} \cup D_{L, \sigma}$; for an external face $\sigma \in \mathcal{E}_{\text {ext }} \cap \mathcal{E}(K), D_{\sigma}$ is just the same volume as $D_{K, \sigma}$.

- MAC discretization - For the MAC scheme, the dual mesh depends on the component of the velocity. For each component, the MAC dual mesh only differs from the RT or CR dual mesh by the choice of the half-diamond cell, which, for $K \in \mathcal{M}$ and $\sigma \in \mathcal{E}(K)$, is now the rectangle or rectangular parallelepiped of basis $\sigma$ and of measure $\left|D_{K, \sigma}\right|=|K| / 2$.

We denote by $\left|D_{\sigma}\right|$ the measure of the dual cell $D_{\sigma}$, and by $\epsilon=D_{\sigma} \mid D_{\sigma^{\prime}}$ the face separating two diamond cells $D_{\sigma}$ and $D_{\sigma^{\prime}}$.

Finally, the hyperbolic systems addressed here will be complemented by an impermeability boundary condition (i.e. $\boldsymbol{u} \cdot \boldsymbol{n}=0$ ). Since the velocity unknowns lie on the boundary (and not inside the cells), this condition is taken into account in the definition of the discrete spaces. To avoid technicalities in the expression of the schemes, we suppose throughout this paper that the boundary is a.e. normal to a coordinate axis, (even in the case of the RT or CR discretizations), which allows to simply set to zero the corresponding velocity unknowns:

$$
\text { for } i=1, \ldots, d, \forall \sigma \in \mathcal{E}_{\mathrm{ext}}^{(i)}, \quad u_{\sigma, i}=0 .
$$

Therefore, there are no degrees of freedom for the velocity on the boundary for the MAC scheme, and there are only $d-1$ degrees of freedom for the CR and RT discretizations, which depend on the orientation of the face of the boundary. In order to be able to write a unique expression of the discrete equations for both MAC and $\mathrm{CR} / \mathrm{RT}$ schemes, we introduce the set of faces $\mathcal{E}_{\mathcal{S}}^{(i)}$ associated to the degrees of freedom of each component of 
the velocity ( $\mathcal{S}$ stands for "scheme"):

$$
\mathcal{E}_{\mathcal{S}}^{(i)}=\mid \begin{aligned}
& \mathcal{E}^{(i)} \backslash \mathcal{E}_{\text {ext }}^{(i)} \text { for the MAC scheme, } \\
& \mathcal{E} \backslash \mathcal{E}_{\text {ext }}^{(i)} \text { for the CR or RT s, }
\end{aligned}
$$

Similarly, we unify the notation for the set of dual faces for both schemes by denoting by $\tilde{\mathcal{E}}_{\mathcal{S}}^{(i)}$ the set of the faces of the dual mesh associated to the $i^{\text {th }}$ component of the velocity. For the CR and RT finite element discretizations, the sets $\tilde{\mathcal{E}}_{\mathcal{S}}^{(i)}, 1 \leq i \leq d$, are the same (before taking into account the boundary conditions, which eliminate some degrees of freedom and thus some dual cells and, finally, some external dual faces). For the MAC scheme, $\tilde{\mathcal{E}}_{\mathcal{S}}^{(i)}$ depends on the component $i$; note that only approximatively $\operatorname{Card}\left(\tilde{\mathcal{E}}_{\mathcal{S}}^{(i)}\right) / d$ faces of $\tilde{\mathcal{E}}_{\mathcal{S}}^{(i)}$ are normal to $e^{(i)}$ (by opposition to what happens for $\mathcal{E}_{\mathcal{S}}^{(i)}$ ), the other ones being normal to another vector of the canonical basis of $\mathbb{R}^{d}$.

Note that general domains can easily be addressed (of course, with the CR or RT discretizations) by redefining, through linear combinations, the degrees of freedom at the external faces, so as to introduce the normal velocity as a new degree of freedom.

\section{The COMPRESSiBle BARotropic Euler EQUATIONS}

We address in this section the so-called barotropic Euler equations, which consist in the following system of partial differential equations:

$$
\begin{aligned}
& \partial_{t} \rho+\operatorname{div}(\rho \boldsymbol{u})=0, \\
& \partial_{t}(\rho \boldsymbol{u})+\operatorname{div}(\rho \boldsymbol{u} \otimes \boldsymbol{u})+\nabla p=0, \\
& p=\wp(\rho)=\rho^{\gamma} .
\end{aligned}
$$

This problem is posed over an open bounded connected subset $\Omega$ of $\mathbb{R}^{d}, 1 \leq d \leq 3$, of boundary $\partial \Omega$, and a finite time interval $(0, T)$. The variable $t$ stands for the time, $\rho, \boldsymbol{u}=\left(u_{1}, \ldots, u_{d}\right)$ and $p$ are the density, velocity and pressure in the flow. The three above equations are respectively the mass balance, the momentum balance and the equation of state of the fluid, which is supposed to take the form $\wp(s)=s^{\gamma}$, where $\gamma \geq 1$ is a coefficient which is specific to the fluid considered. This system must be supplemented by initial conditions for $\rho$ and $\boldsymbol{u}$, denoted by $\rho_{0}$ and $\boldsymbol{u}_{0}$, and we assume $\rho_{0}>0$. It must also be supplemented by a suitable boundary condition, which we suppose to be:

$$
\boldsymbol{u} \cdot \boldsymbol{n}=0,
$$

at any time and a.e. on $\partial \Omega$, where $\boldsymbol{n}$ stands for the normal vector to the boundary.

Let us denote by $E_{k}$ the kinetic energy $E_{k}=\frac{1}{2}|\boldsymbol{u}|^{2}$. Taking the inner product of (1b) by $\boldsymbol{u}$ yields, after formal compositions of partial derivatives and using (1a):

$$
\partial_{t}\left(\rho E_{k}\right)+\operatorname{div}\left(\rho E_{k} \boldsymbol{u}\right)+\nabla p \cdot \boldsymbol{u}=0 .
$$

This relation is referred to as the kinetic energy balance.

Let us now define the function $\mathcal{P}$, from $(0,+\infty)$ to $\mathbb{R}$, as a primitive of $s \mapsto \wp(s) / s^{2}$; this quantity is often called the elastic potential. Let $\mathcal{H}$ be the function defined by $\mathcal{H}(s)=s \mathcal{P}(s), \forall s \in(0,+\infty)$, which, for the specific equation of state used here, yields:

$$
\mathcal{H}(s)=s \mathcal{P}(s)=\left\{\begin{array}{l}
\frac{s^{\gamma}}{\gamma-1} \text { if } \gamma>1, \\
s \ln (s) \text { if } \gamma=1 .
\end{array}\right.
$$


Since $\wp$ is an increasing function, $\mathcal{H}$ is convex. In addition, it may easily be checked that $\rho \mathcal{H}^{\prime}(\rho)-\mathcal{H}(\rho)=\wp(\rho)$. Therefore, by a formal computation, detailed for instance in [10, Appendix], multiplying (1a) by $\mathcal{H}^{\prime}(\rho)$ yields:

$$
\partial_{t}(\mathcal{H}(\rho))+\operatorname{div}(\mathcal{H}(\rho) \boldsymbol{u})+p \operatorname{div}(\boldsymbol{u})=0
$$

Let us denote by $\mathcal{S}$ the quantity $\mathcal{S}=\rho E_{k}+\mathcal{H}(\rho)$. Summing (2) and (4), we get:

$$
\partial_{t} \mathcal{S}+\operatorname{div}((\mathcal{S}+p) \boldsymbol{u})=0 .
$$

In fact, to avoid to invoke unrealistic regularity assumptions, such a computation should be done on the regularized equations (obtained by adding diffusion perturbation terms), and, when making these regularization terms tend to zero, positive measures appear at the left-hand-side of (5), so that we get in the distribution sense:

$$
\partial_{t} \mathcal{S}+\operatorname{div}((\mathcal{S}+p) \boldsymbol{u}) \leq 0 .
$$

The quantity $\mathcal{S}$ is an entropy of the system, and an entropy solution to (1) is thus required to satisfy:

$$
\int_{0}^{T} \int_{\Omega}\left[-\mathcal{S} \partial_{t} \varphi-(\mathcal{S}+p) \boldsymbol{u} \cdot \nabla \varphi\right] \mathrm{d} \boldsymbol{x} \mathrm{d} t-\int_{\Omega} \mathcal{S}_{0} \varphi(\boldsymbol{x}, 0) \mathrm{d} \boldsymbol{x} \leq 0, \quad \forall \varphi \in \mathrm{C}_{c}^{\infty}(\Omega \times[0, T)), \varphi \geq 0,
$$

with $\mathcal{S}_{0}=\frac{1}{2} \rho_{0}\left|\boldsymbol{u}_{0}\right|^{2}+\mathcal{H}\left(\rho_{0}\right)$. Then, since the normal velocity is prescribed to zero at the boundary, integrating (6) over $\Omega$ yields:

$$
\frac{d}{d t} \int_{\Omega}\left[\frac{1}{2} \rho|\boldsymbol{u}|^{2}+\mathcal{H}(\rho)\right] \mathrm{d} \boldsymbol{x} \leq 0
$$

Since $\rho \geq 0$ by (1a) (and the associated initial and boundary conditions) and the function $s \mapsto \mathcal{H}(s)$ is bounded by below and increasing at least for $s$ large enough, Inequality (8) provides an estimate on the solution.

The purpose of this section is to build an explicit scheme for the numerical solution of System (1), and prove the following results:

- a discrete kinetic energy balance (i.e. a discrete analogue of (2)) is established on dual cells, while a discrete potential elastic balance (i.e. a discrete analogue of (4)) is established on primal cells.

Note however that, because of residual terms of unknown sign appearing in the potential elastic balance, contrary to what is obtained for implicit and semi-implicit variants of the present scheme $[5,10]$, these equations do not seem to yield the stability of the scheme (i.e. a discrete global entropy conservation analogue to Equation (8)), at least without supposing drastic limitations of the time step.

- In one space dimension, the limit of any convergent sequence of solutions to the scheme is shown to be a weak solution to the continuous problem, and thus to satisfy the Rankine-Hugoniot conditions.

- Finally, passing to the limit in the discrete kinetic energy and elastic potential balances, such a limit is also shown to satisfy the entropy inequality $(7)$.

\subsection{The scheme}

Let us consider a partition $0=t_{0}<t_{1}<\ldots<t_{N}=T$ of the time interval $(0, T)$, which we suppose uniform for the sake of simplicity, and let $\delta t=t_{n+1}-t_{n}$ for $n=0,1, \ldots, N-1$ be the (constant) time step. We consider 
an explicit-in-time scheme, which reads in its fully discrete form, for $0 \leq n \leq N-1$ :

$$
\begin{aligned}
& \forall K \in \mathcal{M}, \quad \frac{|K|}{\delta t}\left(\rho_{K}^{n+1}-\rho_{K}^{n}\right)+\sum_{\sigma \in \mathcal{E}(K)} F_{K, \sigma}^{n}=0, \\
& \forall K \in \mathcal{M}, \quad p_{K}^{n+1}=\wp\left(\rho_{K}^{n+1}\right)=\left(\rho_{K}^{n+1}\right)^{\gamma},
\end{aligned}
$$

For $1 \leq i \leq d, \forall \sigma \in \mathcal{E}_{\mathcal{S}}^{(i)}$

$$
\frac{\left|D_{\sigma}\right|}{\delta t}\left(\rho_{D_{\sigma}}^{n+1} u_{\sigma, i}^{n+1}-\rho_{D_{\sigma}}^{n} u_{\sigma, i}^{n}\right)+\sum_{\epsilon \in \tilde{\mathcal{E}}\left(D_{\sigma}\right)} F_{\sigma, \epsilon}^{n} u_{\epsilon, i}^{n}+\left|D_{\sigma}\right|(\nabla p)_{\sigma, i}^{n+1}=0,
$$

where the terms introduced for each discrete equation are defined hereafter.

Equation (9a) is obtained by the discretization of the mass balance equation (1a) over the primal mesh, and $F_{K, \sigma}^{n}$ stands for the mass flux across $\sigma$ outward $K$, which, because of the impermeability condition, vanishes on external faces and is given on the internal faces by:

$$
\forall \sigma=K\left|L \in \mathcal{E}_{\text {int }}, \quad F_{K, \sigma}^{n}=\right| \sigma \mid \rho_{\sigma}^{n} u_{K, \sigma}^{n},
$$

where $u_{K, \sigma}^{n}$ is an approximation of the normal velocity to the face $\sigma$ outward $K$. This latter quantity is defined by:

$$
u_{K, \sigma}^{n}=\mid \begin{aligned}
& \boldsymbol{u}_{\sigma}^{n} \cdot \boldsymbol{n}_{K, \sigma} \text { in the CR and RT cases, } \\
& u_{\sigma, i}^{n} \boldsymbol{e}^{(i)} \cdot \boldsymbol{n}_{K, \sigma} \text { for } \sigma \in \mathcal{E}^{(i)} \text { in the MAC case, }
\end{aligned}
$$

where $\boldsymbol{e}^{(i)}$ denotes the $i$-th vector of the orthonormal basis of $\mathbb{R}^{d}$. The density at the face $\sigma=K \mid L$ is approximated by the upwind technique:

$$
\rho_{\sigma}^{n}=\mid \begin{array}{ll}
\rho_{K}^{n} & \text { if } u_{K, \sigma}^{n} \geq 0 \\
\rho_{L}^{n} & \text { otherwise. }
\end{array}
$$

We now turn to the discrete momentum balance (9c), which is obtained by discretizing the momentum balance equation (1b) on the dual cells associated to the faces of the mesh. For the discretization of the time derivative, we must provide a definition for the values $\rho_{D_{\sigma}}^{n+1}$ and $\rho_{D_{\sigma}}^{n}$, which approximate the density on the face $\sigma$ at time $t^{n+1}$ and $t^{n}$ respectively. They are given by the following weighted average:

$$
\text { for } \sigma=K \mid L \in \mathcal{E}_{\text {int }}, \text { for } k=n \text { and } k=n+1, \quad\left|D_{\sigma}\right| \rho_{D_{\sigma}}^{k}=\left|D_{K, \sigma}\right| \rho_{K}^{k}+\left|D_{L, \sigma}\right| \rho_{L}^{k} .
$$

Let us now describe the discretization of the convection term. The first task is to define the discrete mass flux through the dual face $\epsilon$ outward $D_{\sigma}$, denoted by $F_{\sigma, \epsilon}^{n} ;$ the guideline for its construction is that a finite volume discretization of the mass balance equation over the diamond cells, of the form

$$
\forall \sigma \in \mathcal{E}, \quad \frac{\left|D_{\sigma}\right|}{\delta t}\left(\rho_{D_{\sigma}}^{n+1}-\rho_{D_{\sigma}}^{n}\right)+\sum_{\epsilon \in \mathcal{E}\left(D_{\sigma}\right)} F_{\sigma, \epsilon}^{n}=0
$$

must hold in order to be able to derive a discrete kinetic energy balance (see Section 3.2 below). For the MAC scheme, the flux through a dual face which is located on two primal faces is the mean value of the sum of fluxes through the two primal faces, and the flux through a dual face located between two primal faces is again the mean value of the sum of fluxes through the two primal faces [14]. In the case of the CR and RT schemes, for a dual face included in the primal cell $K$, this flux is computed as a linear combination (with constant coefficients, i.e. independent of the cell) of the mass fluxes through the faces of $K$, i.e. the quantities $\left(F_{K, \sigma}^{n}\right)_{\sigma \in \mathcal{E}(K)}$ appearing 
in the discrete mass balance (9a). We refer to $[1,6]$ for a detailed construction of this approximation. Let us remark that a dual face lying on the boundary is then also a primal face, and the flux across this face is zero. Therefore, the values $u_{\epsilon, i}^{n}$ are only needed at the internal dual faces, and we make the upwind choice for their discretization:

$$
\text { for } \epsilon=D_{\sigma}\left|D_{\sigma^{\prime}}, \quad u_{\epsilon, i}^{n}=\right| \begin{array}{ll}
u_{\sigma, i}^{n} & \text { if } F_{\sigma, \epsilon}^{n} \geq 0 \\
u_{\sigma^{\prime}, i}^{n} & \text { otherwise. }
\end{array}
$$

The last term $(\nabla p)_{\sigma, i}^{n+1}$ stands for the $i$-th component of the discrete pressure gradient at the face $\sigma$. The gradient operator is built as the transpose of the divergence operator for the velocity, the discretization of which is based on the primal mesh. Let us denote the divergence of $\boldsymbol{u}^{n+1}$ over $K \in \mathcal{M}$ by $(\operatorname{div} \boldsymbol{u})_{K}^{n+1}$; its natural approximation reads:

$$
\text { for } K \in \mathcal{M}, \quad(\operatorname{div} \boldsymbol{u})_{K}^{n+1}=\frac{1}{|K|} \sum_{\sigma \in \mathcal{E}(K)}|\sigma| u_{K, \sigma}^{n+1} .
$$

Consequently, the components of the pressure gradient are given by:

$$
\text { for } \sigma=K \mid L \in \mathcal{E}_{\mathrm{int}}, \quad(\nabla p)_{\sigma, i}^{n+1}=\frac{|\sigma|}{\left|D_{\sigma}\right|}\left(p_{L}^{n+1}-p_{K}^{n+1}\right) \boldsymbol{n}_{K, \sigma} \cdot \boldsymbol{e}^{(i)},
$$

this expression being derived thanks to the following duality relation with respect to the $\mathrm{L}^{2}$ inner product:

$$
\sum_{K \in \mathcal{M}}|K| p_{K}^{n+1}(\operatorname{div} \boldsymbol{u})_{K}^{n+1}+\sum_{i=1}^{d} \sum_{\sigma \in \mathcal{E}_{\mathcal{S}}^{(i)}}\left|D_{\sigma}\right| u_{\sigma, i}^{n+1}(\nabla p)_{\sigma, i}^{n+1}=0
$$

Note that, because of the impermeability boundary conditions, the discrete gradient is not defined at the external faces.

Finally, the initial approximations for $\rho$ and $\boldsymbol{u}$ are given by the average of the initial conditions $\rho_{0}$ and $\boldsymbol{u}_{0}$ on the primal and dual cells respectively:

$$
\begin{aligned}
& \forall K \in \mathcal{M}, \quad \rho_{K}^{0}=\frac{1}{|K|} \int_{K} \rho_{0}(\boldsymbol{x}) \mathrm{d} \boldsymbol{x}, \\
& \text { for } 1 \leq i \leq d, \forall \sigma \in \mathcal{E}_{\mathcal{S}}^{(i)}, \quad u_{\sigma, i}^{0}=\frac{1}{\left|D_{\sigma}\right|} \int_{D_{\sigma}}\left(\boldsymbol{u}_{0}(\boldsymbol{x})\right)_{i} \mathrm{~d} \boldsymbol{x} .
\end{aligned}
$$

Note that, thanks to the upwind choice in the mass balance equation (9a), if $\rho^{n}$ is positive in (9a), then so is $\rho^{n+1}$ under the following CFL condition:

$$
\delta t \leq \frac{|K|}{\sum_{\sigma \in \mathcal{E}(K)}|\sigma|\left(u_{K, \sigma}^{n}\right)^{+}}
$$

where, for $a \in \mathbb{R}, a^{+}=\max (a, 0)$. Since, by assumption, $\rho_{0}$ is positive, under Condition (20), the discrete density thus remains positive at all times.

\subsection{Kinetic energy balance and elastic potential identity}

We begin by deriving a discrete kinetic energy balance equation, as was already done for the implicit and fractional time step scheme described in [10]. We follow the same lines as in the classical derivation of the kinetic energy balance equation (2) in the continuous setting: the discrete kinetic energy balance is obtained 
by multiplying the ( $i^{\text {th }}$ component of the) momentum balance equation (9c) associated to the face $\sigma$ by $u_{\sigma, i}^{n+1}$ and using the mass balance equation (9a) twice.

Lemma 1 (Discrete kinetic energy balance).

A solution to the system (9) satisfies the following equality, for $1 \leq i \leq d, \sigma \in \mathcal{E}_{\mathcal{S}}^{(i)}$ and $0 \leq n \leq N-1$ :

$$
\frac{1}{2} \frac{\left|D_{\sigma}\right|}{\delta t}\left[\rho_{D_{\sigma}}^{n+1}\left(u_{\sigma, i}^{n+1}\right)^{2}-\rho_{D_{\sigma}}^{n}\left(u_{\sigma, i}^{n}\right)^{2}\right]+\frac{1}{2} \sum_{\epsilon \in \mathcal{E}\left(D_{\sigma}\right)} F_{\sigma, \epsilon}^{n}\left(u_{\epsilon, i}^{n}\right)^{2}+\left|D_{\sigma}\right|(\nabla p)_{\sigma, i}^{n+1} u_{\sigma, i}^{n+1}=-R_{\sigma, i}^{n+1},
$$

with:

$$
\begin{aligned}
R_{\sigma, i}^{n+1}=\frac{1}{2} \frac{\left|D_{\sigma}\right|}{\delta t} \rho_{D_{\sigma}}^{n+1}\left(u_{\sigma, i}^{n+1}-u_{\sigma, i}^{n}\right)^{2}+\frac{1}{2} \sum_{\epsilon=D_{\sigma} \mid D_{\sigma^{\prime}} \in \mathcal{E}\left(D_{\sigma}\right)}\left(F_{\sigma, \epsilon}^{n}\right)^{-}\left(u_{\sigma^{\prime}, i}^{n}-u_{\sigma, i}^{n}\right)^{2} & -\sum_{\epsilon=D_{\sigma} \mid D_{\sigma^{\prime}} \in \mathcal{E}\left(D_{\sigma}\right)}\left(F_{\sigma, \epsilon}^{n}\right)^{-}\left(u_{\sigma^{\prime}, i}^{n}-u_{\sigma, i}^{n}\right)\left(u_{\sigma, i}^{n+1}-u_{\sigma, i}^{n}\right),
\end{aligned}
$$

where, for $a \in \mathbb{R}, a^{-} \geq 0$ is defined by $a^{-}=-\min (a, 0)$.

Equation (21) is a discrete analogue of Equation (2), with an upwind discretization of the convection term. The remainder term $R_{\sigma, i}^{n+1}$ is non-negative under the following CFL condition:

$$
\forall \sigma \in \mathcal{E}_{\mathcal{S}}^{(i)}, \quad \delta t \leq \frac{\left|D_{\sigma}\right| \rho_{D_{\sigma}}^{n+1}}{\sum_{\epsilon \in \mathcal{E}\left(D_{\sigma}\right)}\left(F_{\sigma, \epsilon}^{n}\right)^{-}}
$$

Similarly, the solution to the scheme (9) satisfies a discrete version of the elastic potential identity (4), which we now state.

Lemma 2 (Discrete potential balance). Let $\mathcal{H}$ be defined by (3). A solution to the system (9) satisfies the following equality, for $K \in \mathcal{M}$ and $0 \leq n \leq N-1$ :

$$
\frac{|K|}{\delta t}\left[\mathcal{H}\left(\rho_{K}^{n+1}\right)-\mathcal{H}\left(\rho_{K}^{n}\right)\right]+\sum_{\sigma \in \mathcal{E}(K)}|\sigma| \mathcal{H}\left(\rho_{\sigma}^{n}\right) u_{K, \sigma}^{n}+|K| p_{K}^{n}\left(\operatorname{div} \boldsymbol{u}^{n}\right)_{K}=-R_{K}^{n+1},
$$

with:

$$
\begin{aligned}
R_{K}^{n+1}=\frac{1}{2} \frac{|K|}{\delta t} \mathcal{H}^{\prime \prime}\left(\bar{\rho}_{K, 1}^{n}\right)\left(\rho_{K}^{n+1}-\rho_{K}^{n}\right)^{2}+\frac{1}{2} \sum_{\sigma=K \mid L \in \mathcal{E}(K)}|\sigma|\left(u_{K, \sigma}^{n}\right)^{-} \mathcal{H}^{\prime \prime}\left(\bar{\rho}_{\sigma}^{n}\right)\left(\rho_{K}^{n}-\rho_{L}^{n}\right)^{2} & \\
& +\sum_{\sigma \in \mathcal{E}(K)}|\sigma| u_{K, \sigma}^{n} \mathcal{H}^{\prime \prime}\left(\bar{\rho}_{K, 2}^{n}\right) \rho_{\sigma}^{n}\left(\rho_{K}^{n+1}-\rho_{K}^{n}\right),
\end{aligned}
$$

with $\bar{\rho}_{K, 1}^{n}, \bar{\rho}_{K, 2}^{n} \in\left\|\rho_{K}^{n+1}, \rho_{K}^{n}\right\|$, and $\bar{\rho}_{\sigma}^{n} \in \|\left[\rho_{K}^{n}, \rho_{\sigma}^{n} \|\right.$ for all $\sigma \in \mathcal{E}(K)$, where, for $a, b \in \mathbb{R}$, we denote by $\left.\| a, b\right] \|$ the interval $\| a, b]=\{\theta a+(1-\theta) b, \theta \in[0,1]\}$.

Unfortunately, it does not seem that $R_{K}^{n+1} \geq 0$ in any case, and so we are not able to prove a discrete counterpart of the total entropy estimate (8), which would yield a stability estimate for the scheme. However, under a condition for a time step which is only slightly more restrictive than a CFL-condition, and under some stability assumptions for the solutions to the scheme, we are able to show that this remainder term tends to zero in $\mathrm{L}^{1}(\Omega \times(0, T))$, which allows to conclude, in the $1 \mathrm{D}$ case, that a convergent sequence of solutions satisfies the entropy inequality (7): this is the result stated in Theorem 3.2 below. 


\subsection{Passing to the limit in the scheme (1D case)}

The objective of this section is to show, in the one dimensional case, that if a sequence of solutions is controlled in suitable norms and converges to a limit, this latter necessarily satisfies a (part of the) weak formulation of the continuous problem.

The 1D version of the scheme which is studied in this section may be obtained from Scheme (9) by taking the MAC variant of the scheme, using only one horizontal stripe of grid cells, supposing that the vertical component of the velocity (the degrees of freedom of which are located on the top and bottom boundaries) vanishes, and that the measure of the vertical faces is equal to 1. For the sake of readability, however, we completely rewrite this $1 \mathrm{D}$ scheme, and, to this purpose, we first introduce some adaptations of the notations to the one dimensional case. For any face $\sigma \in \mathcal{E}$, let $x_{\sigma}$ be its abscissa. For any $K \in \mathcal{M}$, we denote by $h_{K}$ its length; when we write $K=\left[\sigma \sigma^{\prime}\right]$, this means that either $K=\left(x_{\sigma}, x_{\sigma^{\prime}}\right)$ or $K=\left(x_{\sigma^{\prime}}, x_{\sigma}\right)$; if we need to specify the order, i.e. $K=\left(x_{\sigma}, x_{\sigma^{\prime}}\right)$ with $x_{\sigma}<x_{\sigma^{\prime}}$, then we write $K=\left[\overrightarrow{\sigma \sigma^{\prime}}\right]$. For an interface $\sigma=K \mid L$ between two cells $K$ and $L$, if we need to specify the order of the cells $K$ and $L$, say $K$ is left of $L$, then we write $\sigma=\overrightarrow{K \mid L}$. With these notations, the explicit scheme (9) may be written as follows in the one dimensional setting:

$$
\begin{aligned}
& \forall K \in \mathcal{M}, \rho_{K}^{0}=\frac{1}{|K|} \int_{K} \rho_{0}(x) \mathrm{d} x, \quad \text { and }, \forall \sigma \in \mathcal{E}_{\text {int }}, u_{\sigma}^{0}=\frac{1}{\left|D_{\sigma}\right|} \int_{D_{\sigma}} u_{0}(x) \mathrm{d} x, \\
& \forall K=\left[\overrightarrow{\sigma \sigma^{\prime}}\right] \in \mathcal{M}, \\
& \qquad \frac{|K|}{\delta t}\left(\rho_{K}^{n+1}-\rho_{K}^{n}\right)+F_{\sigma^{\prime}}^{n}-F_{\sigma}^{n}=0, \\
& \forall K \in \mathcal{M}, \quad p_{K}^{n+1}=\wp\left(\rho_{K}^{n+1}\right)=\left(\rho_{K}^{n+1}\right)^{\gamma}, \\
& \forall \sigma=\overrightarrow{K \mid L} \in \mathcal{E}_{\text {int }}, \quad \frac{\left|D_{\sigma}\right|}{\delta t}\left(\rho_{D_{\sigma}}^{n+1} u_{\sigma}^{n+1}-\rho_{D_{\sigma}}^{n} u_{\sigma}^{n}\right)+F_{L}^{n} u_{L}^{n}-F_{K}^{n} u_{K}^{n}+p_{L}^{n+1}-p_{K}^{n+1}=0 .
\end{aligned}
$$

The mass flux in the discrete mass balance equation is given, for $\sigma \in \mathcal{E}_{\text {int }}$, by $F_{\sigma}^{n}=\rho_{\sigma}^{n} u_{\sigma}^{n}$, where the upwind approximation for the density at the face, $\rho_{\sigma}^{n}$, is defined by (12). In the momentum balance equation, the application of the procedure described in Section 3.1 yields for the density associated to the dual cell $D_{\sigma}$ with $\sigma=K \mid L$ and for the mass fluxes at the dual face located at the center of the mesh $K=\left[\overrightarrow{\sigma \sigma^{\prime}}\right]$ :

$$
\text { for } k=n \text { and } k=n+1, \rho_{D_{\sigma}}^{k}=\frac{1}{2\left|D_{\sigma}\right|}\left(|K| \rho_{K}^{k}+|L| \rho_{L}^{k}\right), \quad F_{K}^{n}=\frac{1}{2}\left(F_{\sigma}^{n}+F_{\sigma^{\prime}}^{n}\right) \text {, }
$$

and the approximation of the velocity at this face is upwind: $u_{K}^{n}=u_{\sigma}^{n}$ if $F_{K}^{n} \geq 0$ and $u_{K}^{n}=u_{\sigma^{\prime}}^{n}$ otherwise.

Let a sequence of discretizations $\left(\mathcal{M}^{(m)}, \delta t^{(m)}\right)_{m \in \mathbb{N}}$ be given. We define the size $h^{(m)}$ of the mesh $\mathcal{M}^{(m)}$ by $h^{(m)}=\sup _{K \in \mathcal{M}^{(m)}} h_{K}$. Let $\rho^{(m)}, p^{(m)}$ and $u^{(m)}$ be the solution given by the scheme $(26)$ with the mesh $\mathcal{M}^{(m)}$ and the time step $\delta t^{(m)}$. To the discrete unknowns, we associate piecewise constant functions on time intervals and on primal or dual meshes, so the density $\rho^{(m)}$, the pressure $p^{(m)}$ and the velocity $u^{(m)}$ are defined almost everywhere on $\Omega \times(0, T)$ by:

$$
\begin{aligned}
\rho^{(m)}(x, t) & =\sum_{n=0}^{N-1} \sum_{K \in \mathcal{M}}\left(\rho^{(m)}\right)_{K}^{n} \mathcal{X}_{K}(x) \mathcal{X}_{[n, n+1)}(t), \quad p^{(m)}(x, t)=\sum_{n=0}^{N-1} \sum_{K \in \mathcal{M}}\left(p^{(m)}\right)_{K}^{n} \mathcal{X}_{K}(x) \mathcal{X}_{[n, n+1)}(t), \\
u^{(m)}(x, t) & =\sum_{n=0}^{N-1} \sum_{\sigma \in \mathcal{E}}\left(u^{(m)}\right)_{\sigma}^{n} \mathcal{X}_{D_{\sigma}}(x) \mathcal{X}_{[n, n+1)}(t)
\end{aligned}
$$


where $\mathcal{X}_{K}, \mathcal{X}_{D_{\sigma}}$ and $\mathcal{X}_{[n, n+1)}$ stand for the characteristic function of the intervals $K, D_{\sigma}$ and $\left[t^{n}, t^{n+1}\right)$ respectively.

For discrete functions $q$ and $v$ defined on the primal and dual mesh, respectively, we define a discrete $\mathrm{L}^{1}((0, T) ; \mathrm{BV}(\Omega))$ norm by:

$$
\|q\|_{\mathcal{T}, x, \mathrm{BV}}=\sum_{n=0}^{N} \delta t \sum_{\sigma=K \mid L \in \mathcal{E}_{\mathrm{int}}}\left|q_{L}^{n}-q_{K}^{n}\right|, \quad\|v\|_{\mathcal{T}, x, \mathrm{BV}}=\sum_{n=0}^{N} \delta t \sum_{\epsilon=D_{\sigma} \mid D_{\sigma^{\prime}} \in \tilde{\mathcal{E}}_{\mathrm{int}}}\left|v_{\sigma^{\prime}}^{n}-v_{\sigma}^{n}\right|,
$$

and a discrete $\mathrm{L}^{1}(\Omega ; \mathrm{BV}((0, T)))$ norm by:

$$
\|q\|_{\mathcal{T}, t, \mathrm{BV}}=\sum_{K \in \mathcal{M}}|K| \sum_{n=0}^{N-1}\left|q_{K}^{n+1}-q_{K}^{n}\right|, \quad\|v\|_{\mathcal{T}, t, \mathrm{BV}}=\sum_{\sigma \in \mathcal{E}}\left|D_{\sigma}\right| \sum_{n=0}^{N-1}\left|v_{\sigma}^{n+1}-v_{\sigma}^{n}\right| .
$$

For the consistency results that we are seeking (Theorems 3.1 and 3.2 below), we have to assume that a sequence of discrete solutions $\left(\rho^{(m)}, p^{(m)}, u^{(m)}\right)_{m \in \mathbb{N}}$ satisfies $\rho^{(m)}>0$ and $p^{(m)}>0, \forall m \in \mathbb{N}$ (which may be a consequence of the fact that the CFL stability condition (20) is satisfied) and is uniformly bounded in $\mathrm{L}^{\infty}(\Omega \times(0, T))^{3}$, i.e.:

$$
0<\left(\rho^{(m)}\right)_{K}^{n} \leq C \text { and } 0<\left(p^{(m)}\right)_{K}^{n} \leq C, \quad \forall K \in \mathcal{M}^{(m)}, \text { for } 0 \leq n \leq N^{(m)}, \forall m \in \mathbb{N},
$$

and:

$$
\left|\left(u^{(m)}\right)_{\sigma}^{n}\right| \leq C, \quad \forall \sigma \in \mathcal{E}^{(m)}, \text { for } 0 \leq n \leq N^{(m)}, \forall m \in \mathbb{N},
$$

where $C$ is a positive real number. Note that, by definition of the initial conditions of the scheme, these inequalities imply that the functions $\rho_{0}$ and $u_{0}$ belong to $\mathrm{L}^{\infty}(\Omega)$. We also have to assume that a sequence of discrete solutions satisfies the following uniform bounds with respect to the discrete BV-norms:

$$
\left\|\rho^{(m)}\right\|_{\mathcal{T}, x, \mathrm{BV}}+\left\|u^{(m)}\right\|_{\mathcal{T}, x, \mathrm{BV}} \leq C, \quad \forall m \in \mathbb{N} .
$$

and:

$$
\left\|\rho^{(m)}\right\|_{\mathcal{T}, t, \mathrm{BV}} \leq C, \quad \forall m \in \mathbb{N}
$$

We are not able to prove the estimates (29)-(32) for the solutions of the scheme; however, such inequalities are satisfied by the "interpolation" (for instance, by taking the cell average) of the solution to a Riemann problem, and are observed in computations (of course, as far as possible, i.e. with a limited sequence of meshes and time steps).

A weak solution to the continuous problem satisfies, for any $\varphi \in \mathrm{C}_{c}^{\infty}(\Omega \times[0, T))$ :

$$
\begin{aligned}
& -\int_{0}^{T} \int_{\Omega}\left[\rho \partial_{t} \varphi+\rho u \partial_{x} \varphi\right] \mathrm{d} x \mathrm{~d} t-\int_{\Omega} \rho_{0}(x) \varphi(x, 0) \mathrm{d} x=0, \\
& -\int_{0}^{T} \int_{\Omega}\left[\rho u \partial_{t} \varphi+\left(\rho u^{2}+p\right) \partial_{x} \varphi\right] \mathrm{d} x \mathrm{~d} t-\int_{\Omega} \rho_{0}(x) u_{0}(x) \varphi(x, 0) \mathrm{d} x=0, \\
& p=\rho^{\gamma} .
\end{aligned}
$$

Note that these relations are not sufficient to define a weak solution to the problem, since they do not imply anything about the boundary conditions. However, they allow to derive the Rankine-Hugoniot conditions; hence if we show that they are satisfied by the limit of a sequence of solutions to the discrete problem, this implies, loosely speaking, that the scheme computes correct shocks (i.e. shocks where the jumps of the unknowns and of the fluxes are linked to the shock speed by Rankine-Hugoniot conditions). This is the result we are seeking and which we now state. 
Theorem 3.1 (Consistency of the one-dimensional explicit scheme, barotropic case).

Let $\Omega$ be an open bounded interval of $\mathbb{R}$. We suppose that the initial data satisfies $\rho_{0} \in \mathrm{L}^{\infty}(\Omega)$ and $u_{0} \in \mathrm{L}^{\infty}(\Omega)$. Let $\left(\mathcal{M}^{(m)}, \delta t^{(m)}\right)_{m \in \mathbb{N}}$ be a sequence of discretizations such that both the time step $\delta t^{(m)}$ and the size $h^{(m)}$ of the mesh $\mathcal{M}^{(m)}$ tend to zero as $m \rightarrow+\infty$, and let $\left(\rho^{(m)}, p^{(m)}, u^{(m)}\right)_{m \in \mathbb{N}}$ be the corresponding sequence of solutions. We suppose that this sequence satisfies the estimates (29)-(31) and converges in $\mathrm{L}^{p}(\Omega \times(0, T))^{3}$, for $1 \leq p<\infty$, to $(\bar{\rho}, \bar{p}, \bar{u}) \in \mathrm{L}^{\infty}(\Omega \times(0, T))^{3}$.

Then the limit $(\bar{\rho}, \bar{p}, \bar{u})$ satisfies the system (33).

Main ideas of the proof - It is clear that with the assumed convergence for the sequence of solutions, the limit satisfies the equation of state. The proof of this theorem is thus obtained by passing to the limit in the scheme, first for the mass balance equation and then for the momentum balance equation. This is performed by considering a smooth function $\varphi$ over the domain $\Omega$, defining its interpolate $\varphi_{\mathcal{M}}$ over the cells and its interpolate $\varphi_{\mathcal{E}}$ over the edges. Then one first multiplies the discrete mass balance equation (26b) by the value $\varphi_{K}^{n+1}$ of the interpolate of $\varphi$ on $K$ at $t^{n+1}$, sum over the cells and time steps, and, introducing the discrete time and space derivatives of $\varphi$ and noting that they tend uniformly to the continuous time and space derivatives of $\varphi$, pass to the limit on all terms to recover (33a). Similarly, one multiplies the discrete momentum equation (26d) by the value $\varphi_{\sigma}^{n+1}$ of $\varphi$ on $\sigma$ at $t^{n+1}$, sum over the edges and time steps, and again pass to the limit on all terms to recover $(33 \mathrm{~b})$. The details of this proof may be found in $[15,20]$.

We now turn to the entropy condition (7). To this purpose, we need to introduce the following additional condition for a regular sequence of discretizations:

$$
\lim _{m \rightarrow \infty} \frac{\delta t^{(m)}}{\min _{K \in \mathcal{M}^{(m)}} h_{K}}=0
$$

Note that this condition is slightly more restrictive than a standard CFL condition. Then we are in position to state the following consistency result.

Theorem 3.2 (Entropy consistency, barotropic case). Under the assumptions of Theorem 3.1, if we suppose in addition that the considered sequence of discretizations satisfies (34), the sequence of solutions satisfies (32) and that, for $\gamma<2$, the sequence $\left(1 / \rho^{(m)}\right)_{m \in \mathbb{N}}$ is uniformly bounded in $\mathrm{L}^{\infty}(\Omega \times(0, T))$, then the limit $(\bar{\rho}, \bar{p}, \bar{u})$ satisfies the entropy condition (7).

Main ideas of the proof - The proof of this theorem is again based on a passage to the limit in discrete equations, namely the discrete kinetic balance equation (21) and the elastic potential balance (24). This computation is very close to the proof of consistency of the scheme for the full Euler equations with the total energy balance, i.e. the proof of Theorem 4.1 below. We refer to $[15,20]$ for the details of this computation.

Remark 3.3 (On BV-stability assumptions).

The proof of Theorem 3.1 in $[15,20]$ shows that the scheme is consistent under a BV-stability assumption much weaker than (31), namely:

$$
\lim _{m \rightarrow+\infty} h^{(m)}\left[\left\|\rho^{(m)}\right\|_{\mathcal{T}, x, \mathrm{BV}}+\left\|u^{(m)}\right\|_{\mathcal{T}, x, \mathrm{BV}}\right]=0 .
$$

The situation is completely different when proving that the limit of convergent sequences is an entropy solution (i.e. when proving Theorem 3.2 , see once again $[15,20]$ ), since the stability condition which is actually used in the proof is:

$$
\lim _{m \rightarrow+\infty} \frac{\delta t^{(m)}}{\min _{K \in \mathcal{M}^{(m)}} h_{K}}\left\|\rho^{(m)}\right\|_{\mathcal{T}, t, \mathrm{BV}}=0
$$




\section{The Full Euler equations}

Let us now turn to the full compressible Euler equations, which read:

$$
\begin{aligned}
& \partial_{t} \rho+\operatorname{div}(\rho \boldsymbol{u})=0, \\
& \partial_{t}(\rho \boldsymbol{u})+\operatorname{div}(\rho \boldsymbol{u} \otimes \boldsymbol{u})+\nabla p=0, \\
& \partial_{t}(\rho E)+\operatorname{div}(\rho E \boldsymbol{u})+\operatorname{div}(p \boldsymbol{u})=0, \\
& p=(\gamma-1) \rho e, \quad E=\frac{1}{2}|\boldsymbol{u}|^{2}+e,
\end{aligned}
$$

where $E$ and $e$ are the total and internal energy respectively, and $\gamma>1$ is a coefficient specific to the considered fluid. System (35) is complemented by initial conditions for $\rho, e$ and $\boldsymbol{u}$, denoted by $\rho_{0}, e_{0}$ and $\boldsymbol{u}_{0}$ respectively, with $\rho_{0}>0$ and $e_{0}>0$, and by a boundary condition which we suppose to be $\boldsymbol{u} \cdot \boldsymbol{n}=0$ at any time and a.e. on $\partial \Omega$.

Let us suppose that the solution is regular. Subtracting the kinetic energy balance equation (2) from the total energy balance (35c), we obtain the internal energy balance equation:

$$
\partial_{t}(\rho e)+\operatorname{div}(\rho e \boldsymbol{u})+p \operatorname{div}(\boldsymbol{u})=0 .
$$

Since,

- thanks to the mass balance equation, the first two terms in the left-hand side of (36) may be recast as a transport operator: $\partial_{t}(\rho e)+\operatorname{div}(\rho e \boldsymbol{u})=\rho\left[\partial_{t} e+\boldsymbol{u} \cdot \boldsymbol{\nabla} e\right]$,

- and, from the equation of state, the pressure vanishes when $e=0$,

this equation implies, if $e \geq 0$ at $t=0$ and with suitable boundary conditions, that $e$ remains non-negative at all times.

We wish to build an explicit version of the staggered implicit and semi-implicit schemes that have already been studied for the Euler equations [11]. As already mentioned in [11], discretizing (36) instead of the total energy balance (35c) presents two advantages:

- $\quad$ first, it avoids the space discretization of the total energy, which is rather unnatural for staggered schemes since the degrees of freedom for the velocity and the scalar variables are not collocated,

- second, a suitable discretization of (36) may yield, "by construction" of the scheme, the positivity of the internal energy.

However, for solutions with shocks, Equation (36) is not equivalent to (35c); more precisely speaking, at the locations of shocks, positive measures should appear at the right-hand side of Equation (36). Discretizing (36) instead of (35c) may thus yield a scheme which does not compute the correct weak discontinuous solutions; in particular, the numerical solutions may present (smeared) shocks which do not satisfy the Rankine-Hugoniot conditions associated to (35c). The essential result of this section is to provide solutions to circumvent this problem. To this purpose, we closely mimic the above performed formal computation:

- we start from the discrete kinetic energy balance (21), and remark that the residual terms at the righthand side do not tend to zero with the space and time steps (they are the discrete manifestations of the above mentioned measures),

- we thus compensate these residual terms by corrective terms in the internal energy balance.

We provide a theoretical justification of this process by showing that, in the $1 \mathrm{D}$ case, if the scheme is stable and converges to a limit (in a sense to be defined), this limit satisfies a weak form of (35c) which implies the correct Rankine-Hugoniot conditions. 


\subsection{The scheme}

With the same notations as in Section 3.1, we consider an explicit-in-time numerical scheme for the discretization of the Euler equations, i.e. System (35). In its fully discrete form, this scheme reads, for $0 \leq n \leq N-1$ :

$$
\begin{aligned}
& \forall K \in \mathcal{M}, \quad \frac{|K|}{\delta t}\left(\rho_{K}^{n+1}-\rho_{K}^{n}\right)+\sum_{\sigma \in \mathcal{E}(K)} F_{K, \sigma}^{n}=0, \\
& \forall K \in \mathcal{M}, \quad \frac{|K|}{\delta t}\left(\rho_{K}^{n+1} e_{K}^{n+1}-\rho_{K}^{n} e_{K}^{n}\right)+\sum_{\sigma \in \mathcal{E}(K)} F_{K, \sigma}^{n} e_{\sigma}^{n}+|K| p_{K}^{n}(\operatorname{div} \boldsymbol{u})_{K}^{n}=S_{K}^{n}, \\
& \forall K \in \mathcal{M}, \quad p_{K}^{n+1}=(\gamma-1) \rho_{K}^{n+1} e_{K}^{n+1},
\end{aligned}
$$$$
\text { For } 1 \leq i \leq d, \forall \sigma \in \mathcal{E}_{\mathcal{S}}^{(i)}
$$$$
\frac{\left|D_{\sigma}\right|}{\delta t}\left(\rho_{D_{\sigma}}^{n+1} u_{\sigma, i}^{n+1}-\rho_{D_{\sigma}}^{n} u_{\sigma, i}^{n}\right)+\sum_{\epsilon \in \tilde{\mathcal{E}}\left(D_{\sigma}\right)} F_{\sigma, \epsilon}^{n} u_{\epsilon, i}^{n}+\left|D_{\sigma}\right|(\nabla p)_{\sigma, i}^{n+1}=0 .
$$

Equations (37a) and (37d) are the same as the discrete mass and momentum balance equations (9a) and (9c) of the barotropic model, and are described in Section 3.1. Equation (37b) is an approximation of the internal energy balance over the primal cell $K$. The positivity of the convection operator is ensured if we use an upwinding technique for this term [17]:

$$
\text { for } \sigma=K\left|L \in \mathcal{E}_{\text {int }}, \quad e_{\sigma}^{n}=\right| \begin{array}{ll}
e_{K}^{n} & \text { if } F_{K, \sigma}^{n} \geq 0 \\
e_{L}^{n} & \text { otherwise. }
\end{array}
$$

The discrete divergence of the velocity, $(\operatorname{div} \boldsymbol{u})_{K}^{n}$, is defined by (16) and the discrete pressure gradient by $(17)$, so that the discrete gradient and divergence operators are dual with respect to the $\mathrm{L}^{2}$ inner product, as stated in Relation (18). The right-hand side, $S_{K}^{n}$, is derived using consistency arguments in the next section.

Finally, the initial approximations for $\rho$ and $\boldsymbol{u}$ are given by (19) and the initial condition for $e$ is the following one:

$$
\forall K \in \mathcal{M}, \quad e_{K}^{0}=\frac{1}{|K|} \int_{K} e_{0}(\boldsymbol{x}) \mathrm{d} \boldsymbol{x} .
$$

Since, by assumption, $e_{0}>0$, the (discrete) initial condition for the internal energy is positive. Using standard arguments, thanks to the fact that, in the third term of $(37 \mathrm{~b}), p_{K}^{n}$ is proportional to $e_{K}^{n}$ (precisely speaking, $\left.p_{K}^{n}=(\gamma-1) \rho_{K}^{n} e_{K}^{n}\right)$, we prove that the internal energy remains positive at all times assuming (20) and the following additional CFL condition:

$$
\delta t \leq \frac{|K|}{\gamma \sum_{\sigma \in \mathcal{E}(K)}|\sigma|\left(u_{K, \sigma}^{n}\right)^{+}}, \quad \forall K \in \mathcal{M}
$$

provided that $S_{K}^{n}$ is positive, which is the case under another CFL condition (see Inequality (42) in the next section).

\subsection{The discrete kinetic energy balance equation and the corrective source terms}

By Lemma 1, we know that a discrete kinetic balance holds, with, at the right-hand side, some residual terms. The next step is now to define corrective terms in the internal energy balance, with the aim to recover a 
consistent discretization of the total energy balance. The first idea to do this could be just to sum the (discrete) kinetic energy balance with the internal energy balance: it is indeed possible for a collocated discretization. But here, we face the fact that the kinetic energy balance is associated to the dual mesh, while the internal energy balance is discretized on the primal mesh. The way to circumvent this difficulty is to remark that we do not really need a discrete total energy balance; in fact, we only need to recover (a weak form of) this equation when the mesh and time steps tend to zero. To this purpose, we choose the quantities $\left(S_{K}^{n}\right)$ in such a way as to somewhat compensate the terms $\left(R_{\sigma, i}^{n}\right)$ given by $(22)$ :

$$
\begin{aligned}
\forall K \in \mathcal{M}, S_{K}^{n+1}=\sum_{i=1}^{d} S_{K, i}^{n+1} \text { with } S_{K, i}^{n+1}=\frac{1}{2} \rho_{K}^{n+1} \sum_{\substack{\sigma \in \mathcal{E}(K) \cap \mathcal{E}_{\mathcal{S}}^{(i)}\\
}} \frac{\left|D_{K, \sigma}\right|}{\delta t}\left(u_{\sigma, i}^{n+1}-u_{\sigma, i}^{n}\right)^{2} \\
+\sum_{\substack{\epsilon \in \tilde{\mathcal{E}}_{\mathcal{S}}^{(i)}, \epsilon \cap \bar{K} \neq \emptyset, \epsilon=D_{\sigma} \mid D_{\sigma^{\prime}}, F_{\sigma, \epsilon}^{n} \leq 0}} \alpha_{K, \epsilon}\left[\frac{\left|F_{\sigma, \epsilon}^{n}\right|}{2}\left(u_{\sigma, i}^{n}-u_{\sigma^{\prime}, i}^{n}\right)^{2}+F_{\sigma, \epsilon}^{n}\left(u_{\sigma, i}^{n+1}-u_{\sigma, i}^{n}\right)\left(u_{\sigma^{\prime}, i}^{n}-u_{\sigma, i}^{n}\right)\right] .
\end{aligned}
$$

The coefficient $\alpha_{K, \epsilon}$ is fixed to 1 if the face $\epsilon$ is included in $K$, and this is the only situation to consider for the RT and CR discretizations; these faces appear twice when summing (22) over the faces $\sigma$ of the primal mesh, once with $F_{\sigma, \epsilon}^{n} \geq 0$ and once with $F_{\sigma, \epsilon}^{n}<0$ and they appear only once when summing $S_{K}^{n+1}$ over the cells $K$ of the primal mesh; the terms in (22) coming from the convection term are nonzero only if $F_{\sigma, \epsilon}^{n}<0$, and, in the last of these two terms, the upwind and downwind faces play a different role, which is apparent in the above definition of the summation indexes $\left(\epsilon\right.$ is oriented in such a way that $D_{\sigma}$ is the downwind dual cell). For the MAC scheme, some dual faces are included in the primal cells, and for these, we set $\alpha_{K, \epsilon}=1$, but some lie on their boundary; these latter faces again appear twice when summing (22) over the faces $\sigma$ of the primal mesh (again once with $F_{\sigma, \epsilon}^{n}<0$ and once with $F_{\sigma, \epsilon}^{n} \geq 0$ ), but they appear four times when summing $S_{K}^{n+1}$ over the cells $K$ of the primal mesh. However, the remainders $\left(R_{\sigma, i}^{n}\right)$ must be distributed only between the two downstream primal cells (i.e. the cells in which the flow through $\epsilon$ is entering), to allow to prove that the corrective terms $\left(S_{K}^{n}\right)$ are non-negative. For such a dual face $\epsilon=D_{\sigma} \mid D_{\sigma^{\prime}}$, since, by notation, $D_{\sigma}$ stands for the dual cell downwind $\epsilon, \alpha_{K, \epsilon}$ is given by:

$$
\alpha_{K, \epsilon}=\frac{1}{2} \text { if } \sigma \in \mathcal{E}(K), \text { and } \alpha_{K, \epsilon}=0 \text { otherwise. }
$$

The expression of the terms $\left(S_{K}^{n+1}\right)_{K \in \mathcal{M}}$ is justified by the passage to the limit in the scheme (for a onedimensional problem) performed in Section 4.3. However, we can already note that, as explained above:

$$
\sum_{K \in \mathcal{M}} S_{K}^{n+1}-\sum_{i=1}^{d} \sum_{\sigma \in \mathcal{E}_{\mathcal{S}}^{(i)}} R_{\sigma, i}^{n+1}=0
$$

Indeed, the first part of $S_{K, i}^{n+1}$, thanks to the expression (27) of the density at the face $\rho_{D_{\sigma}}^{n+1}$, results from a dispatching of the first part of the residual over the two adjacent cells:

$$
\frac{1}{2} \frac{\left|D_{\sigma}\right|}{\delta t} \rho_{D_{\sigma}}^{n+1}\left(u_{\sigma, i}^{n+1}-u_{\sigma, i}^{n}\right)^{2}=\underbrace{\frac{1}{2} \frac{\left|D_{K, \sigma}\right|}{\delta t} \rho_{K}^{n+1}\left(u_{\sigma, i}^{n+1}-u_{\sigma, i}^{n}\right)^{2}}_{\text {affected to K }}+\underbrace{\frac{1}{2} \frac{\left|D_{L, \sigma}\right|}{\delta t} \rho_{L}^{n+1}\left(u_{\sigma, i}^{n+1}-u_{\sigma, i}^{n}\right)^{2}}_{\text {affected to L }} .
$$

The same argument holds for the terms associated to the dual faces, which explains, in particular, the definition of the coefficients $\alpha_{K, \epsilon}$. The scheme thus conserves the discrete equivalent of the integral of the total energy over the computational domain. 
Using Young's inequality, we obtain that $S_{K, i}^{n+1}$ in non-negative provided that the following CFL condition holds for $0 \leq n \leq N-1$, for all $K \in \mathcal{M}$ and $\sigma \in \mathcal{E}(K)$ :

$$
\delta t \leq \frac{\left|D_{K, \sigma}\right| \rho_{K}^{n+1}}{\sum_{\epsilon \in \tilde{\mathcal{E}}\left(D_{\sigma}\right), \epsilon \cap \bar{K} \neq \emptyset} \alpha_{K, \epsilon}\left(F_{\sigma, \epsilon}^{n}\right)^{-}} .
$$

Under the conditions (39) and (42), the solution given by the scheme thus satisfies $\rho>0$ and $e>0$, and so $p>0$ by the equation of state. The conservation by the scheme of the integral of the total energy over the computational domain thus yields a control on the solution.

\subsection{Passing to the limit in the scheme (1D case)}

The objective of this section is to show, in the one dimensional case, that, if a sequence of solutions is controlled in suitable norms and converges to a limit, this latter necessarily satisfies a weak formulation of the continuous problem. With the notations of Section 3.3, the one-dimensional version of the explicit scheme (37) reads:

$$
\begin{aligned}
& \forall K \in \mathcal{M}, \quad \rho_{K}^{0}=\frac{1}{|K|} \int_{K} \rho_{0}(x) \mathrm{d} x, \quad e_{K}^{0}=\frac{1}{|K|} \int_{K} e_{0}(x) \mathrm{d} x, \\
& \forall \sigma \in \mathcal{E}_{\text {int }}, \quad u_{\sigma}^{0}=\frac{1}{\left|D_{\sigma}\right|} \int_{D_{\sigma}} u_{0}(x) \mathrm{d} x, \\
& \forall K=\left[\overrightarrow{\sigma \sigma^{\prime}}\right] \in \mathcal{M}, \quad \frac{|K|}{\delta t}\left(\rho_{K}^{n+1}-\rho_{K}^{n}\right)+F_{\sigma^{\prime}}^{n}-F_{\sigma}^{n}=0, \\
& \forall K=\left[\overrightarrow{\sigma \sigma^{\prime}}\right] \in \mathcal{M}, \quad \frac{|K|}{\delta t}\left(\rho_{K}^{n+1} e_{K}^{n+1}-\rho_{K}^{n} e_{K}^{n}\right)+F_{\sigma^{\prime}}^{n} e_{\sigma^{\prime}}^{n}-F_{\sigma}^{n} e_{\sigma}^{n}+p_{K}^{n}\left(u_{\sigma^{\prime}}^{n}-u_{\sigma}^{n}\right)=S_{K}^{n}, \\
& \forall K \in \mathcal{M}, \quad p_{K}^{n+1}=(\gamma-1) \rho_{K}^{n+1} e_{K}^{n+1} . \\
& \forall \sigma=\overrightarrow{K \mid L} \in \mathcal{E}_{\text {int }}, \quad \frac{\left|D_{\sigma}\right|}{\delta t}\left(\rho_{D_{\sigma}}^{n+1} u_{\sigma}^{n+1}-\rho_{D_{\sigma}}^{n} u_{\sigma}^{n}\right)+F_{L}^{n} u_{L}^{n}-F_{K}^{n} u_{K}^{n}+p_{L}^{n+1}-p_{K}^{n+1}=0 .
\end{aligned}
$$

In the convection terms of the internal energy balance, the approximation for $e_{\sigma}^{n}$ is upwind with respect to $F_{\sigma}^{n}$ (i.e., for $\sigma=\overrightarrow{K \mid L} \in \mathcal{E}_{\text {int }}, e_{\sigma}^{n}=e_{K}^{n}$ if $F_{\sigma}^{n} \geq 0$ and $e_{\sigma}^{n}=e_{L}^{n}$ otherwise). The corrective term $S_{K}^{n}$ reads, $\forall K=\left[\sigma^{\prime} \rightarrow \sigma\right]:$

$$
S_{K}^{n+1}=\frac{|K|}{4 \delta t} \rho_{K}^{n}\left[\left(u_{\sigma}^{n+1}-u_{\sigma}^{n}\right)^{2}+\left(u_{\sigma^{\prime}}^{n+1}-u_{\sigma^{\prime}}^{n}\right)^{2}\right]+\frac{\left|F_{K}^{n}\right|}{2}\left(u_{\sigma}^{n}-u_{\sigma^{\prime}}^{n}\right)^{2}-\left|F_{K}^{n}\right|\left(u_{\sigma}^{n}-u_{\sigma}^{n}\right)\left(u_{\sigma^{\prime}}^{n}-u_{\sigma}^{n}\right),
$$

where the notation $K=\left[\sigma^{\prime} \rightarrow \sigma\right]$ means that the flow goes from $\sigma^{\prime}$ to $\sigma$ (i.e., if $F_{K}^{n} \geq 0, K=\left[\overrightarrow{\sigma^{\prime} \sigma}\right]$ and, if $\left.F_{K}^{n} \leq 0, K=\left[\overrightarrow{\sigma \sigma^{\prime}}\right]\right)$.

To the discrete unknowns, we associate piecewise constant functions on time intervals and on primal or dual cells, so the density $\rho$, the pressure $p$, and the velocity $u$ are defined almost everywhere on $\Omega \times(0, T)$ by $(28)$, and $e$ is defined a.e. by:

$$
e(x, t)=\sum_{n=0}^{N-1} \sum_{K \in \mathcal{M}} e_{K}^{n} \mathcal{X}_{K}(x) \mathcal{X}_{[n, n+1)}(t)
$$

For the consistency result that we are seeking (Theorem 4.1 below), we have to assume that a sequence of discrete solutions $\left(\rho^{(m)}, p^{(m)}, e^{(m)}, u^{(m)}\right)_{m \in \mathbb{N}}$ satisfies $\rho^{(m)}>0, p^{(m)}>0$, and $e^{(m)}>0, \forall m \in \mathbb{N}$ (which may be 
a consequence of the fact that the CFL stability conditions (20), (39) and (42) are satisfied), and is uniformly bounded in $\mathrm{L}^{\infty}(\Omega \times(0, T))^{4}$, i.e.:

$$
0<\left(\rho^{(m)}\right)_{K}^{n} \leq C, 0<\left(p^{(m)}\right)_{K}^{n} \leq C \text { and } 0<\left(e^{(m)}\right)_{K}^{n} \leq C, \quad \forall K \in \mathcal{M}^{(m)}, \text { for } 0 \leq n \leq N^{(m)}, \forall m \in \mathbb{N},
$$

and:

$$
\left|\left(u^{(m)}\right)_{\sigma}^{n}\right| \leq C, \quad \forall \sigma \in \mathcal{E}^{(m)}, \text { for } 0 \leq n \leq N^{(m)}, \forall m \in \mathbb{N},
$$

where $C$ stands for a positive real number. We also have to assume that a sequence of discrete solutions satisfies the following uniform bounds with respect to the discrete BV-norms:

$$
\left\|\rho^{(m)}\right\|_{\mathcal{T}, x, \mathrm{BV}}+\left\|p^{(m)}\right\|_{\mathcal{T}, x, \mathrm{BV}}+\left\|e^{(m)}\right\|_{\mathcal{T}, x, \mathrm{BV}}+\left\|u^{(m)}\right\|_{\mathcal{T}, x, \mathrm{BV}} \leq C, \quad \forall m \in \mathbb{N}
$$

and:

$$
\left\|u^{(m)}\right\|_{\mathcal{T}, t, \mathrm{BV}} \leq C, \quad \forall m \in \mathbb{N}
$$

A weak solution to the continuous problem satisfies, for any $\varphi \in \mathrm{C}_{c}^{\infty}(\Omega \times[0, T))$ :

$$
\begin{aligned}
& -\int_{\Omega \times(0, T)}\left[\rho \partial_{t} \varphi+\rho u \partial_{x} \varphi\right] \mathrm{d} x \mathrm{~d} t-\int_{\Omega} \rho_{0}(x) \varphi(x, 0) \mathrm{d} x=0 \\
& -\int_{\Omega \times(0, T)}\left[\rho u \partial_{t} \varphi+\left(\rho u^{2}+p\right) \partial_{x} \varphi\right] \mathrm{d} x \mathrm{~d} t-\int_{\Omega} \rho_{0}(x) u_{0}(x) \varphi(x, 0) \mathrm{d} x=0 \\
& -\int_{\Omega \times(0, T)}\left[\rho E \partial_{t} \varphi+(\rho E+p) u \partial_{x} \varphi\right] \mathrm{d} x \mathrm{~d} t-\int_{\Omega} \rho_{0}(x) E_{0}(x) \varphi(x, 0) \mathrm{d} x=0, \\
& p=(\gamma-1) \rho e, \quad E=\frac{1}{2} u^{2}+e, \quad E_{0}=\frac{1}{2} u_{0}^{2}+e_{0} .
\end{aligned}
$$

As in the barotropic case, these relations are not sufficient to define a weak solution to the problem, but they allow to derive the Rankine-Hugoniot conditions; therefore, if we show that they are satisfied by the limit of a sequence of solutions to the discrete problem, we can expect that the scheme computes correct shocks; this is stated in the following theorem.

Theorem 4.1 (Consistency of the one-dimensional explicit scheme, Euler case).

Let $\Omega$ be an open bounded interval of $\mathbb{R}$. We suppose that the initial data satisfies $\rho_{0} \in \mathrm{L}^{\infty}(\Omega), p_{0} \in \mathrm{BV}(\Omega)$, $e_{0} \in \mathrm{L}^{\infty}(\Omega)$ and $u_{0} \in \mathrm{L}^{\infty}(\Omega)$. Let $\left(\mathcal{M}^{(m)}, \delta t^{(m)}\right)_{m \in \mathbb{N}}$ be a sequence of discretizations such that both the time step $\delta t^{(m)}$ and the size $h^{(m)}$ of the mesh $\mathcal{M}^{(m)}$ tend to zero as $m \rightarrow+\infty$. Let $\left(\rho^{(m)}, p^{(m)}, e^{(m)}, u^{(m)}\right)_{m \in \mathbb{N}}$ be the corresponding sequence of solutions. We suppose that this sequence satisfies the estimates (45)-(48) and converges in $\mathrm{L}^{r}(\Omega \times(0, T))^{4}$, for $1 \leq r<\infty$, to $(\bar{\rho}, \bar{p}, \bar{e}, \bar{u}) \in \mathrm{L}^{\infty}(\Omega \times(0, T))^{4}$.

Then the limit $(\bar{\rho}, \bar{p}, \bar{e}, \bar{u})$ satisfies the system (49).

Main ideas of the proof - The proof that the limit $(\bar{\rho}, \bar{p}, \bar{u})$ satisfies (49a) and (49b) is the same as in the barotropic case. In addition, the fact that $(\bar{\rho}, \bar{p}, \bar{e})$ satisfies the equation of state is straightforward, in view of the supposed convergence. We thus only need to prove that $(\bar{\rho}, \bar{p}, \bar{e}, \bar{u})$ satisfies $(49 \mathrm{c})$. In order to do so, the technique is the same as for proving the entropy inequality in the barotropic case. For a given smooth function $\varphi$, on one hand, we multiply the discrete kinetic energy equation (2) by $\delta t \varphi_{\sigma}^{n+1}$, where $\varphi_{\sigma}^{n}$ is an interpolate of $\varphi$ at the face $\sigma$ and at $t^{n+1}$, and sum over the faces and the time steps. On the other hand, we multiply the discrete internal energy equation (36) by $\delta t \varphi_{K}^{n+1}$, where $\varphi_{K}^{n+1}$ is an interpolate of $\varphi$ on $K$ at $t^{n+1}$, and sum over the primal cells and the time steps. Finally, summing the two obtained relations, a bit of algebra allows to conclude; we refer to $[16,20]$ for the detailed computation. 
Remark 4.2 (On BV-stability assumptions).

The proofs of the consistency theorems 3.1 and 4.1 (see [15] and [16], respectively) show that the scheme is consistent under a BV-stability assumption much weaker than (47)-(48), namely:

$$
\lim _{m \rightarrow+\infty}\left(h^{(m)}+\delta t^{(m)}\right)\left[\left\|\rho^{(m)}\right\|_{\mathcal{T}, x, \mathrm{BV}}+\left\|p^{(m)}\right\|_{\mathcal{T}, x, \mathrm{BV}}+\left\|e^{(m)}\right\|_{\mathcal{T}, x, \mathrm{BV}}+\left\|u^{(m)}\right\|_{\mathcal{T}, x, \mathrm{BV}}+\left\|u^{(m)}\right\|_{\mathcal{T}, t, \mathrm{BV}}\right]=0
$$

\section{Some Numerical RESUlts}

We test here the proposed schemes on some one dimensional model problems. More extensive tests can be found in $[15,16,20]$.

\subsection{The barotropic case}

In this section, we give some numerical results for the barotropic equations, taking $p=\rho^{2}$ as equation of state. Note that, in this case, the system is equivalent (up to a constant proportionality coefficient in the equation of state) to the shallow water equations, replacing $\rho$ by the water height $h$. We test the explicit scheme studied above, which we denote by $\rho \rightarrow p \rightarrow u$, since the pressure is updated before solving the momentum balance equation; we also test the "naive" explicit scheme obtained by evaluating all terms other than the time derivative at time $t^{n}$, which we denote by $\rho \rightarrow u \rightarrow p$ (the pressure is now updated, in this variant, after the computation of the velocity rather than after the computation of the density).

We consider a one-dimensional Riemann problem with the following values for the left and right states: $\rho_{L}=1, \rho_{R}=10, u_{L}=5$ and $u_{R}=7.5$, which yields a solution with a left shock and a right rarefaction wave. We plot the computed density and velocity at time $T=0.025$ on Figures 2 and 3 respectively. These results have been obtain with $h=0.001$ and $\delta t=h / 12$ (the maximum velocity and sound speed computed from the analytical solution being $u_{\max }=7.5$ and $c_{\max } \simeq 4.5$, respectively). From these results, it appears clearly that the so-called $\rho \rightarrow u \rightarrow p$ scheme generates discontinuities in the rarefaction wave, and further experiments show that this phenomenon is not cured by a reduction of the time and space steps; this seems to be connected to the fact that, for this variant, we cannot prove that the limits of converging sequences satisfy the entropy condition (and they probably do not). When trying to do so, in our proof and from a purely technical point of view, the trouble comes from the fact that the pressure gradient term which appears in the kinetic energy balance reads $\boldsymbol{u}^{n+1} \boldsymbol{\nabla} p^{n}$ and it seems difficult to make the counterpart with the corresponding time levels $\left(\right.$ i.e. $\left.p^{n} \operatorname{div}\left(\boldsymbol{u}^{n+1}\right)\right)$ appear in the elastic potential balance, starting from a mass balance with a convection term written with $\boldsymbol{u}^{n}$; hence a discretization of the momentum balance equation with an updated pressure gradient term $\nabla p^{n+1}$, and thus the inversion of steps in the algorithm, to get the "reference variant" proposed in this paper. This latter scheme seems to converge to the right solution, and it is confirmed by a numerical convergence study letting the space and time steps tend to zero, which show an approximatively first-order rate of convergence.

\subsection{The full Euler equations}

Let us now turn to the full Euler equations. We use the test case referred as Test 4 in [22, Chapter 4], which is a Riemann problem with the following initial states: $\rho_{L}=\rho_{R}=1, u_{L}=u_{R}=0, p_{L}=0.01$ and $p_{R}=100$. Here again, we test the scheme that was analyzed in Section 4 , which we denote by $\rho \rightarrow e \rightarrow p \rightarrow u$ (the order in which we update the unknowns) and compare it to the "naive" scheme, denoted by $\rho \rightarrow u \rightarrow e \rightarrow p$, obtained by discretizing the Euler equations (35) in the corresponding order.

The density, velocity and pressure obtained at $T=0.035$ with $h=0.001$ and $\delta t=h / 30$ (to be related to a maximal celerity of waves close to 18), together with the analytical solution, are plotted on Figures 4,5 and 6 respectively. For the naive scheme, the same behaviour as for the barotropic case (i.e. the presence of discontinuities in the rarefaction wave) is observed, while, once again, the reference variant of the algorithm yields correct results. However, the diffusive character of the scheme is evidenced at the contact discontinuity; the implementation of a more accurate discretization, based on a MUSCL-like technique, is underway. We also 


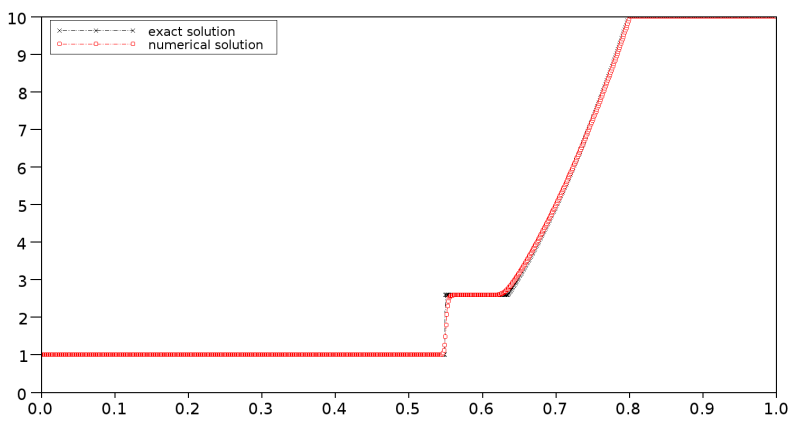

(A) $\rho \rightarrow p \rightarrow u$

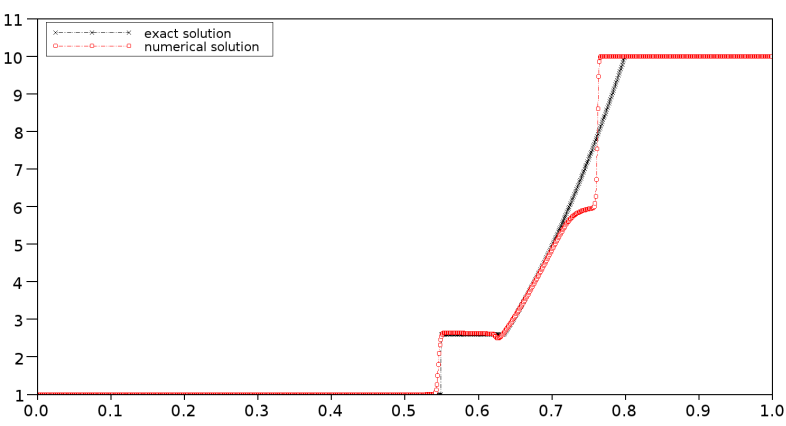

(в) $\rho \rightarrow u \rightarrow p$

Figure 2. Barotropic Euler equations, density

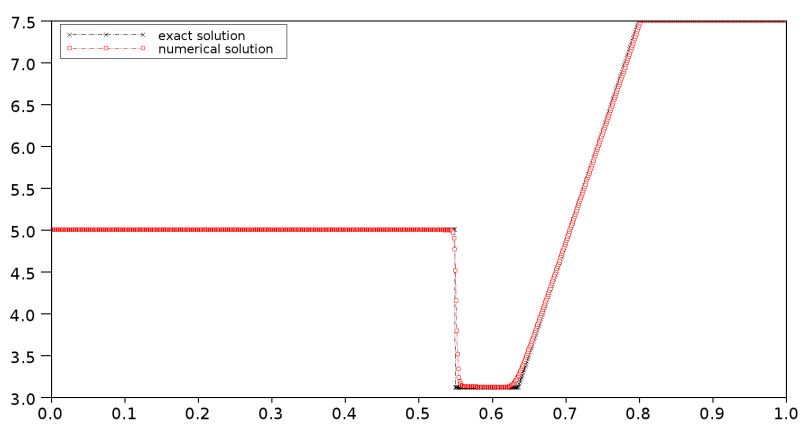

(A) $\rho \rightarrow p \rightarrow u$

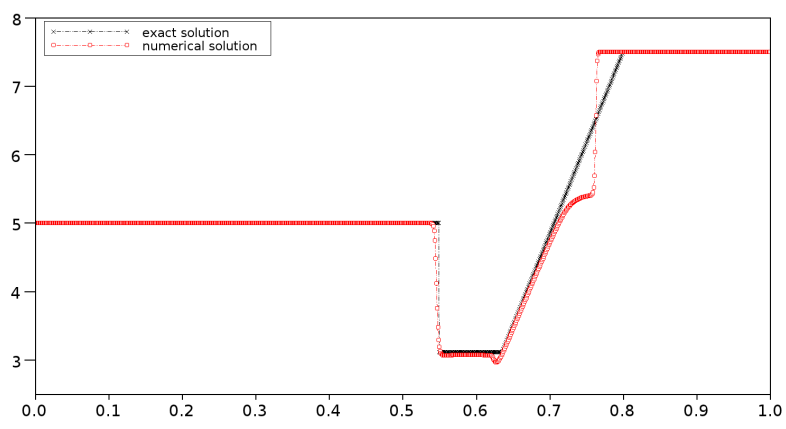

(в) $\rho \rightarrow u \rightarrow p$

FIGURE 3. Barotropic Euler equations, velocity

observe that the scheme keeps the velocity and pressure constant through the contact discontinuity; this may be checked directly from the expression of the discrete balance equations (precisely speaking, one may prove that, if $p^{n}$ and $u^{n}$ are constant, so are $p^{n+1}$ and $u^{n+1}$ ). The order of the scheme is numerically found to be 1 for the variables with no jump at the contact discontinuity (these are $p$ and $u$ ) and $1 / 2$ for those with a jump, namely $\rho$ and $e$.

In addition, we also tested the scheme obtained by neglecting the corrective terms $\left(S_{K}\right)_{K \in \mathcal{M}}$ in the internal energy balance; results (not plotted here) seem to show that this scheme does converge, but toward a limit which is clearly not a weak solution to the Euler equations (in particular, with jumps which do not satisfy the Rankine-Hugoniot conditions).

\section{REFERENCES}

[1] G. Ansanay-Alex, F. Babik, J.-C. Latché, and D. Vola. An L ${ }^{2}$-stable approximation of the Navier-Stokes convection operator for low-order non-conforming finite elements. International Journal for Numerical Methods in Fluids, 66:555-580, 2011.

[2] F. Bouchut. Nonlinear Stability of finite volume methods for hyperbolic conservation laws. Birkhauser, 2004. 


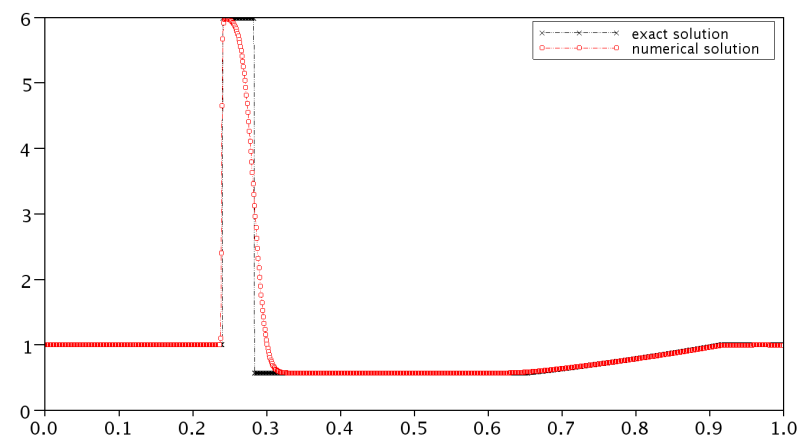

(A) $\rho \rightarrow e \rightarrow p \rightarrow u$

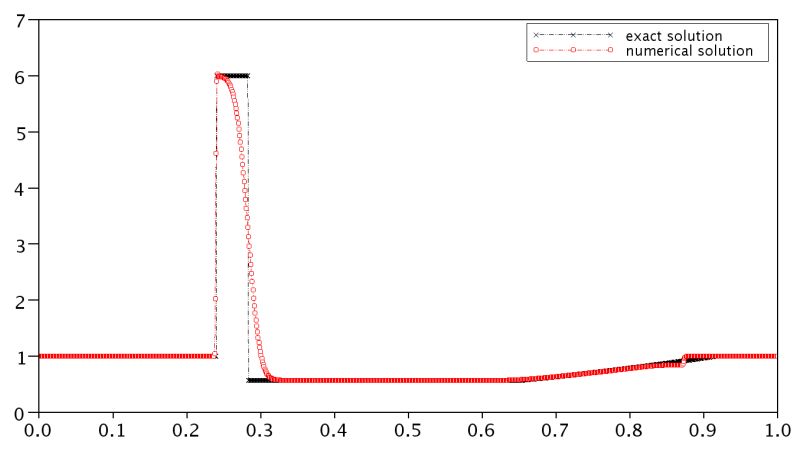

(в) $\rho \rightarrow u \rightarrow e \rightarrow p$

FiguRE 4. Full Euler equations, density

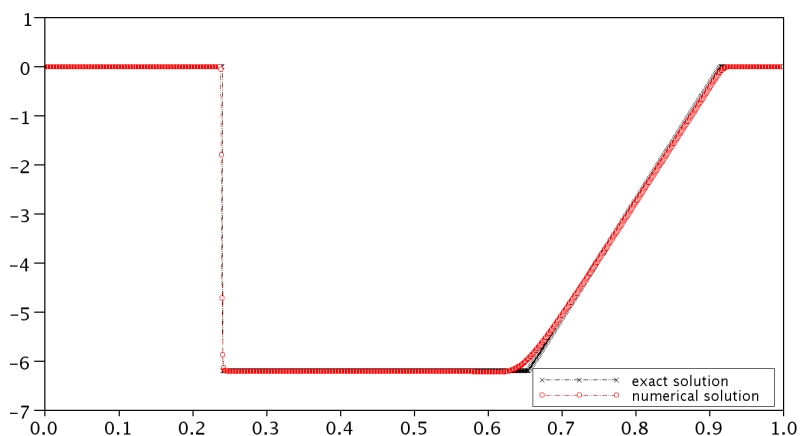

(A) $\rho \rightarrow e \rightarrow p \rightarrow u$

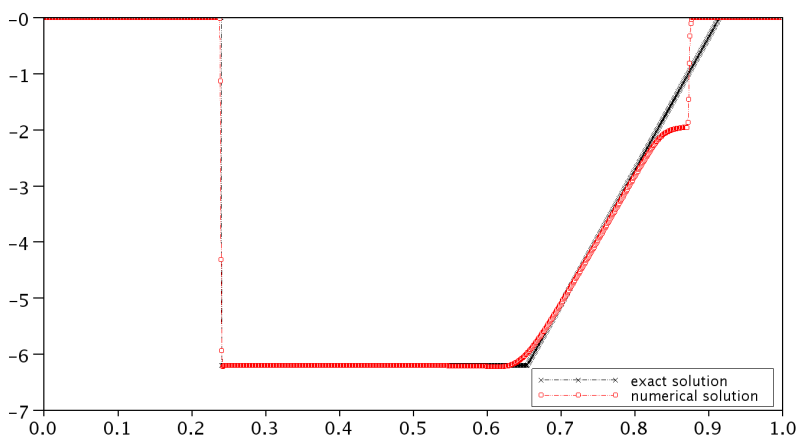

(в) $\rho \rightarrow u \rightarrow e \rightarrow p$

Figure 5. Full Euler equations, velocity

[3] P. G. Ciarlet. Basic error estimates for elliptic problems. In P. Ciarlet and J.L. Lions, editors, Handbook of Numerical Analysis, Volume II, pages 17-351. North Holland, 1991.

[4] M. Crouzeix and P.A. Raviart. Conforming and nonconforming finite element methods for solving the stationary Stokes equations. RAIRO Série Rouge, 7:33-75, 1973.

[5] T. Gallouët, L. Gastaldo, R. Herbin, and J.-C. Latché. An unconditionally stable pressure correction scheme for compressible barotropic Navier-Stokes equations. Mathematical Modelling and Numerical Analysis, 42:303-331, 2008.

[6] L. Gastaldo, R. Herbin, W. Kheriji, C. Lapuerta, and J.-C. Latché. Staggered discretizations, pressure correction schemes and all speed barotropic flows. In Finite Volumes for Complex Applications VI - Problems and Perspectives - Prague, Czech Republic, volume 2, pages 39-56, 2011.

[7] L. Gastaldo, R. Herbin, and J.-C. Latché. A discretization of phase mass balance in fractional step algorithms for the drift-flux model. IMA Journal of Numerical Analysis, 31:116-146, 2011.

[8] F.H. Harlow and A.A. Amsden. A numerical fluid dynamics calculation method for all flow speeds. Journal of Computational Physics, 8:197-213, 1971.

[9] F.H. Harlow and J.E. Welsh. Numerical calculation of time-dependent viscous incompressible flow of fluid with free surface. Physics of Fluids, 8:2182-2189, 1965.

[10] R. Herbin, W. Kheriji, and J.-C. Latché. Consistent semi-implicit staggered schemes for compressible flows - Part I: the barotropic Euler equations. submitted, 2012. 


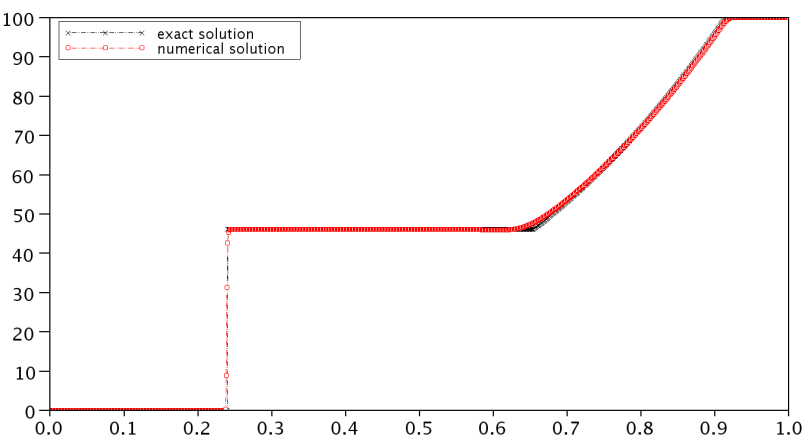

(A) $\rho \rightarrow e \rightarrow p \rightarrow u$

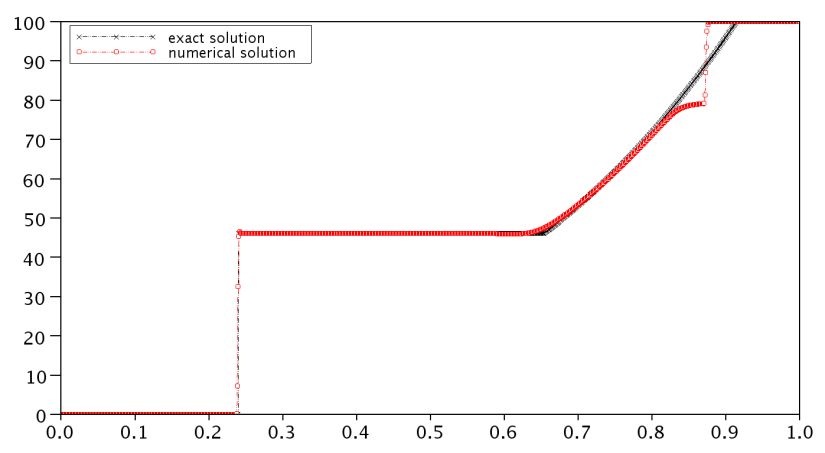

(в) $\rho \rightarrow u \rightarrow e \rightarrow p$

Figure 6. Full Euler equations, pressure

[11] R. Herbin, W. Kheriji, and J.-C. Latché. Consistent semi-implicit staggered schemes for compressible flows - Part II: the Euler equations. submitted, 2012.

[12] R. Herbin, W. Kheriji, and J.-C. Latché. Staggered schemes for all speed flows. Esaim Proc., 2012.

[13] R. Herbin, W. Kheriji, and J.-C. Latché. An unconditionally stable pressure correction scheme for compressible Navier-Stokes equations. submitted, 2013.

[14] R. Herbin and J.-C. Latché. Kinetic energy control in the MAC discretization of the compressible Navier-Stokes equations. International Journal of Finites Volumes, 7, 2010.

[15] R. Herbin, J.-C. Latché, and T.T. Nguyen. Consistent explicit staggered schemes for compressible flows - Part I: the barotropic Euler equations. submitted, 2013.

[16] R. Herbin, J.-C. Latché, and T.T. Nguyen. Consistent explicit staggered schemes for compressible flows - Part II: the Euler equations. submitted, 2013.

[17] B. Larrouturou. How to preserve the mass fractions positivity when computing compressible multi-component flows. Journal of Computational Physics, 95:59-84, 1991.

[18] M.-S. Liou. A sequel to AUSM, part II:AUSM+-up. Journal of Computational Physics, 214:137-170, 2006.

[19] M.-S. Liou and C.J. Steffen. A new flux splitting scheme. Journal of Computational Physics, 107:23-39, 1993.

[20] T.T. Nguyen. Explicit staggered numerical schemes for compressible flows. PhD thesis, Université d'Aix-Marseille, 2013.

[21] R. Rannacher and S. Turek. Simple nonconforming quadrilateral Stokes element. Numerical Methods for Partial Differential Equations, 8:97-111, 1992.

[22] E. Toro. Riemann solvers and numerical methods for fluid dynamics - A practical introduction (third edition). Springer, 2009. 